\title{
Maximal Singularity-Free Orientation Subregions Associated with Initial Parallel Manipulator Configuration
}

\author{
Luis Garcia *, ${ }^{*}$ and Alexandre Campos ${ }^{+}$ \\ Computational Mechanics Laboratory, Universidade do Estado de Santa Catarina, Joinville-SC 89219-710, Brazil; \\ alexandre.campos@udesc.br \\ * Correspondence: luisgarciagonzalez.10@gmail.com \\ + These authors contributed equally to this work.
}

Received: 16 July 2018; Accepted: 11 September 2018; Published: 18 September 2018

\begin{abstract}
Reduced workspace is the main parallel robot disadvantage. It is generally due to the robot configuration, mainly the platform orientation constraint, the present work intends to find the maximum sphere within the orientation workspace, i.e., the singularity-free orientation regions. These regions are related to the platform orientation through Roll-Pitch-Yaw angles. Therefore, an optimization genetic algorithm is used to determine the initial platform orientation corresponding to the largest sphere volume. In this algorithm, the geometrical parameters and the direct and inverse singularities are the optimization constraints. The geometrical constraints may be studied using vectorial analysis. The reciprocity property from screw theory is implemented to analyze the direct and inverse kinematic. In this work it is used a methodology to verify the singularity closeness measure associated with direct kinematic. This measure is related to the rate of work done by each leg upon the platform twist. To determine how close is the parallel robot to a direct singularity a index value is proposed. It is considered that the passive joints reachable regions may be limited by a cone, whereby the cone symmetric axis is the same than the passive joint axis. In the optimization problem, the sphere volume, i.e., the maximal angular displacement of the moving platform around any axis is the objective function. Thus, the genetic algorithm individuals explore all feasible regions looking for an optimal solution.
\end{abstract}

Keywords: parallel robot; workspace; singularities; optimization

\section{Introduction}

According to their structural topology, a parallel robot consists of two platforms (fixed and moving), connected through serial (open-loops) kinematic chains [1]. The fixed platform is called base and the moving platform is called platform. The parallel robot presents advantages in terms of dynamic properties, load carrying capacity, high accuracy and stiffness, which are widely used in the industry [2]. However, the parallel robot orientation-workspace is reduced if it is compared with the serial robot orientation workspace. This disadvantage is analyzed aiming to improve the robot orientation capacity using the kinematics and geometrical robot parameters. Each of these parameters are related to orientation-workspace by means of screw theory used to numerically optimize the parallel robot orientation range. The present work intends to maximize the sphere within the orientation workspace.

For kinematic analysis, synthesis and application planning, the parallel robot workspace is an important property. A general workspace is a six dimensional volumetric object and its characterization is complex due to its complicated geometry. Therefore, to replace it by a convex shape, i.e., sphere could be of great importance in kinematic optimization [3]. Different methods have been presented related 
to workspace with a sphere. In [4], a procedure to obtain the maximal area ellipse and the maximal volume ellipsoid within the feasible workspace of the cable driven parallel robot wrench, using convex optimization is proposed. In this procedure, the workspace boundaries equations are relaxed by means of Weierstrass and Chebyshev approximation theorems. The wrench feasible workspace is the set of postures of the moving platform for which the cables can balance any wrench for a given set of wrenches. To approximate the workspace to a convex geometry (sphere) it is necessary to describe correctly all the singularity free regions.

In [5], the parallel robot design is optimized through the workspace analysis using the tilt and torsion angles to define the platform orientation. For that purpose, an optimization procedure to obtain the higher workspace volume is implemented for a initial platform location. Such procedure consists in two parts. First, the workspace volume is determined by considering the platform initial location to the coordinates subset $x, y$ and torsion angle $\varphi$. Subsequently, the same analysis is done considering the remaining coordinates subset $(z, \theta, \phi)$. In [6], a mathematical commercial software is used to get the reachable workspace for a 3-DOF PUS\&S parallel robot used in the large fuselage or wing assembly of aircraft manufacturing. The geometrical restriction and kinematic singularities are considered using screw theory and Tilt-and-Torsion angle method to obtain a proper design based on the workspace representation. The Tilt-and-Torsion angle method is implemented to describe the platform orientation and the screw theory to describe the actuated joint behaviour related to the platform motion.

In parallel robots, workspace may be limited by singular configurations, which may be inverse, direct or combined. Inverse singularity occurs when the robot loses one or more degrees of freedom. Direct singularity occurs when the platform gains one or more degrees of freedom, whose identification is a complex task [1]. Combined singularities appear when the robot falls in an inverse and direct singularity. Several methods are proposed to analyze the robot singularities simultaneously. In [7], the linear decomposition is used to approach the architecture parameter effects on the nature of the singularity loci. An algorithm based on analytical expression for the Jacobian matrix determinant is implemented. This is possible using two different approaches: linear decomposition and cofactor expansion. The first relates the architecture parameters with the robot singularities loci, while the second approach reduces significantly the determinant computation. In [8], a method to analyze singularities geometrically using Grassmann-Cayley algebra (GCA) is introduced. There, the actuation forces and constraint moments are applied to the platform through their legs. Therefore, a parallel robot is analyzed, relating leg configurations to the geometrical conditions, these conditions are associated with the six Plücker vectors constituting the inverse Jacobian matrix rows. Additionally, in [9], the Grassmann-Cayley algebra analysis is implemented to obtain the geometrical conditions of singularities using the screw theory. Then the Grassmann-Cayley algebra and the associated superbracket decomposition are used. These methods are implemented to determine the jacobian matrix condition, in which the screw axes for each leg are contained.

In [10], the singularity locus of a Gough-Stewart platform is analyzed through a surface over the field of rational functions on the group of rotations. In the generic biplanar case, the parallel planes family cut the surface in a linear pencil of conics and the rotational parametrization are uniform for all generic orientations. They are determined from the geometrical surface properties.

The optimization algorithms implemented in these problems are mainly based on stochastic concepts due to the parallel robot analysis complexity [11]. These algorithms follow certain characteristics and behavior of biological, molecular, swarm of insects, and neurobiological systems. The main advantage of these algorithms are that they do not require derivatives [12].

In [13], a 2-DOF medical parallel robot kinematics is studied to obtain the maximal workspace area by means of genetic algorithms (GAs). The optimization algorithm explores all feasible parallel robot configurations to find the optimal solution. In this optimization problem the kinematics singularities are the constraints, while the geometrical parameters are the input data to calculate the optimal singularity-free cylindrical workspace and to determine continuous singularity-free zones. In [14], an algorithm is developed to detect the optimal singularity-free cylindrical workspace ranging from 
an initial orientation angle in platform to any prescribed orientation. In this case, the algorithm is implemented in a 3-RPR planar robot, using robot structural parameters as constraints. The particle Swarm Optimization (PSO) algorithm is used to determinate the closest point on the singularity surface to the axis of the cylinder.

This paper is organized as follows: Section 2 briefly recalls the parallel robot definitions and provides systems of equations characterizing the platform motions. These equation systems are based on the escrew theory. The mobility contraints are introduced in Section 3 to describe the platfom motions completely. Parallel robot contraints are divided into kinematical and geometrical. The first are associated with platform orientation and the second to the parallel robot physical parameters. These constraints are considered the workspace boundaries which are analyzed in Section 4 to describe the feasible regions related to the platform orientations. Section 5 proposes an algorithm to locate the initial platform orientation bounded by the higher sphere within the orientation workspace. Its performance is demostrated with the Stewart-Gough (S-G) parallel robot. Section 6 summarizes the main conclusions and suggests subjects for future works.

\section{Kinematic of S-G}

The spatial parallel robot used in the proposed analysis is a S-G platform with 6-DoF (see Figure 1). The moving platform, from now on called platform, and the fixed base are connected by six extensible (prismatic joint) and identical legs. These connectors consist of one universal and one spherical joint at points $A_{i}$ and $B_{i}$, respectively. Therefore each leg is a UPS (universal, prismatic and spherical) kinematic chain, where the underline indicates the actuated joint. It should be noted that for the S-G platform the prismatic joint is actuated.

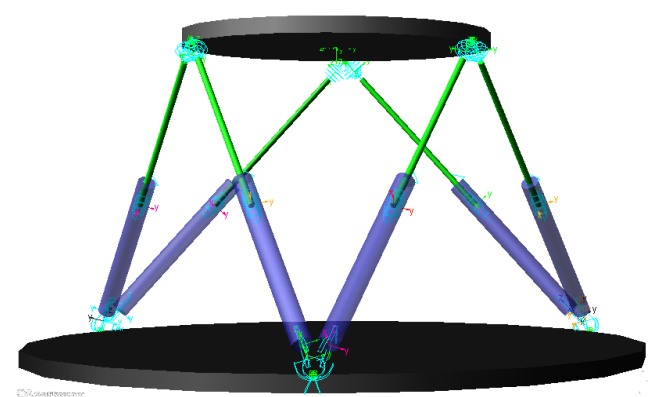

Figure 1. Stewart-Gough Platform in MSC ADAMS/View.

Let $0_{1}, 0_{2}, 0_{3}$ be the fixed coordinate systems which are located at centroid 0 on the base, at the centroid $P$, on the platform and at the ith point $A_{i}$, in each limb, respectively. In the coordinate system $0_{3}$, the $z_{i}$ axis is located in direction from $A_{i}$ to $B_{i}$, the $y_{i}$-axis is parallel to the cross product of two unit vectors along the $z_{i}$ and $z$ axes, the $x_{i}$-axis is defined by the right-hand rule.

Universal joints at point $A_{i}$ and spherical joints at point $B_{i}$ lie on the plane $x-y$ and $u-v$ respectively. Due to this, it is possible to define two position vectors, the first vector $a_{i}$ describes the Ai position on the fixed base frame $0_{1}$, while the second vector ${ }^{2} b_{i}$ describes the $B_{i}$ position on the platform frame, which may be described as:

$$
a_{i}=\left|\begin{array}{c}
R \cos \left(\theta_{i}\right) \\
R \sin \left(\theta_{i}\right) \\
0
\end{array}\right|,{ }^{2} b_{i}=\left|\begin{array}{c}
r \cos \left(\theta_{i}+\theta_{0}\right) \\
r \sin \left(\theta_{i}+\theta_{0}\right) \\
0
\end{array}\right| ; i=1 \text { to } 3
$$

where $\theta_{0}=60$ and the vector $p=[X, Y, Z]$ describes the platform location in the fixed base frame (see Figure 2). It is composed by $X, Y$ and $Z$, which are the centroid coordinates related to the base. For mapping between $x-y-z$ and $u-v-w$ frames, the platform orientation is described by means of the RPY angles. 


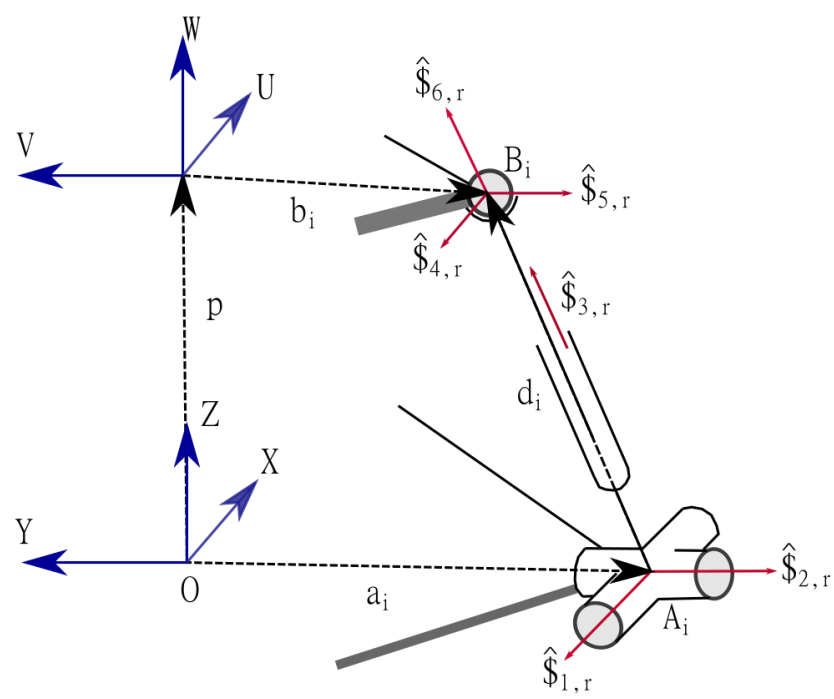

Figure 2. Stewart-gough Platform ith leg configuration.

\subsection{Screw and Reciprocal Screw}

It is possible to express the rigid body infinitesimal displacement as a translation and a rotation around a unique axis. This combined motion is called screw displacement [15]. The screw theory is a mathematical tool commonly implemented in the parallel robot analysis. The latter may be applied to indicate the position and orientation of a spatial body, it may conveniently be represented by two three-dimensional vectors [16]:

$$
\hat{\$}=\left|\begin{array}{c}
\hat{s} \\
s_{0} \times \hat{s}+\lambda \hat{s}
\end{array}\right|
$$

The unit vector $\hat{s}$ is along the screw axis and $s_{0}$ is the position vector between the origin point on the frame and any point on the screw axis. $s_{0} \times s$ may be defined as the geometric moment of the screw axis about the origin reference frame. The pitch $\lambda$ is the relation between the linear and angular displacement, $\lambda=d / \theta$. Therefore, for a prismatic joint $\lambda=\infty$ and for a revolute joint $\lambda=0$ [17]. Thus, for the manipulator shown in Figure 1 the unitary screw associated with the actuated joint in each kinematic chain is a unit screw with $\infty$-pitch $(\lambda=\infty)$, it is wrriten as $\hat{\$}=|0 ; s|$. The screw direction may be expressed as a vectorial operation:

$$
\hat{s}=\frac{p+b_{i}-a_{i}}{\left\|A B_{i}\right\|}
$$

where $b_{i}={ }^{1} R_{2}{ }^{2} b_{i}$ and $a_{i}$ denote the spherical and universal joint positions with respect to the fixed frame in the $i$ th leg. The vector $p$ was previously explained and $\left\|A B_{i}\right\|$ is the leg length,

$$
\left\|A B_{i}\right\|=\left|p+b_{i}-a_{i}\right| .
$$

Thus, for the parallel manipulator $i$-th leg scheme shown in Figure 2, the relationship between joint screw axes and platform screws may be extended as [18]:

$$
\$_{p}=\dot{\theta}_{(1, i)} \hat{\phi}_{1, i}+\dot{\theta}_{(2, i)} \hat{\phi}_{2, i}+\dot{d}_{(3, i)} \hat{\phi}_{3, i}+\dot{\theta}_{(4, i)} \hat{\$}_{4, i}+\dot{\theta}_{(5, i)} \hat{\phi}_{5, i}+\dot{\theta}_{(6, i)} \hat{\phi}_{6, i} \text {. }
$$

There is a screw that only performs work on the actuated joint (prismatic joint), which is called reciprocal [19]. For the $i$-th kinematic chain show in Figure 2 the reciprocal screw $\left(\hat{\$}_{r}\right)$ passes through 
the universl joint center and the spherical joint center [20]. Thus, the unit reciprocal direction may be described by Equation (3), i.e., $s_{r}=s$,

$$
\hat{\$}_{r}=\left|\begin{array}{c}
\hat{s}_{r} \\
S_{r 0} \times \hat{s}_{r}
\end{array}\right| .
$$

\subsection{Screw-Based Jacobian}

Premultiplying Equation (5) by the reciprocal screw $\hat{\$}$

$$
\hat{\$}_{r, i}^{T} \hat{\phi}_{p}=\hat{\$}_{r, i}^{T} \hat{\phi}_{3, i} \dot{d}_{(3, i)}
$$

where

$$
\hat{\$}_{r, i}^{T} \hat{\phi}_{3, i}=1
$$

and the unitary screw transpose is

$$
\hat{\$}_{r, i}^{T}=\left|\begin{array}{ll}
s_{r 0} \times s_{r} & s_{r}
\end{array}\right|
$$

Extending the analysis to the other legs and writing in matrix form

$$
\left|\begin{array}{cc}
s_{r 0,1} \times s_{r, 1} & s_{r, 1} \\
\vdots & \vdots \\
s_{r 0,6} \times s_{r, 6} & s_{r, 6}
\end{array}\right| \$_{p}=|I|\left|\begin{array}{c}
\dot{d}_{(3,1)} \\
\vdots \\
\dot{d}_{(3,6)}
\end{array}\right|
$$

or

$$
J_{x}\left|\$_{p}\right|=J_{q}|\dot{d}| .
$$

$J_{q}$ and $J_{x}$ are the Jacobians related to inverse and direct kinematics, respectively. Thus, the velocities present in the platform and the actuated joints are related by a unified Jacobian defined as $J=J_{x}^{-1} J_{q}[21]$.

\section{Constraints Formulation}

In the proposed analysis the constraints are composed by kinematical (parallel robot singularities) and geometrical restrictions. The first are the particular platform locations where the PMs may gain, direct singularities, or lose, inverse singularities, degrees of freedom. Thus, the singularity analysis determines the conditions under which singularities occur and how to avoid them [22]. The second, geometrical constraints, are due to the parallel robot structural nature. The PMs mobility may be limited by constraints associated with the physical robot parameters.

\subsection{Kinematic Singularities Analysis}

Acording to [22] there are three types of kinematic singularities, each with a different physical interpretation. If the matrix $J_{q}$ is singular, the singularity is inverse. If the matrix $J_{x}$ is singular, the singularity is direct. In addition, if two matrices $J_{q}$ and $J_{x}$ become singular, the singularity is mixed.

\subsubsection{Inverse Singularity}

The inverse kinematic singularity type is caused due the leg serial nature. It may occur at a workspace boundary or on internal boundaries within the workspace regions [23]. This singularity occurs when the determinant of $J_{q}$ goes to zero [24].

$$
\operatorname{Det}\left(J_{q}\right)=0 .
$$


It means that there is a zero platform twist for non zero actuated joint velocities [25]. In other words, for a given non-null velocity, the platform remains immobile, i.e., $\dot{q}$ represents the non empty null space of the singular matrix $J_{q}$ [26]. For the present study case the parallel robot is inverse singularity free due to the fact that $J_{q}$ matrix is an identity matrix, see Equation (10). Hence the robot mobility is only constrained by the direct singularity and the geometrical constraints.

\subsubsection{Direct Singularity}

Direct singularity is more complex than inverse singularity because it appears inside the workspace. In this case, the platform is not controllable, which means that the parallel robot may gain one or more degrees of freedom. The parallel robot is in direct singularity when the matrix is singular, i.e.,

$$
\operatorname{Det}\left(J_{x}\right)=0,
$$

where the platform twist is the non empty null space. That is, even if the actuated joints are locked the platform may move in some directions [1] . It means that the parallel robot cannot withstand forces in some directions [25]. The direct singularity occurs only in parallel robots and it is difficult to define. Aiming at identifying the direct singularity, Voglewede [27] proposes a methodology called power measure, or work measure, using the screw theory. This technique measures the closeness of direct singularities by means of an optimization problem. This problem consideres the objective function as

$$
F=\sum_{i=1}^{n}\left(\hat{\$}_{r, i}^{T} \circ \$\right)^{2}
$$

where $\$_{r}$ is a wrench acting upon the platform, $\$$ is the platform twist and $n$ is the total number of limbs. The Equation (14) is interpreted as the sum of the square work done by each leg upon the platform motion. Hence, Equation (14) may be rewritten as

$$
F=\left[\begin{array}{llll}
W_{1} & W_{2} & \cdots & W_{n}
\end{array}\right]\left[\begin{array}{c}
W_{1} \\
W_{2} \\
\vdots \\
W_{n}
\end{array}\right]
$$

with

$$
[W]=J_{x}[\$]
$$

Thus, F may be written in the quadratic form [28]

$$
F=\$^{T} J_{x}^{T} J_{x} \$=\$^{T} G \$
$$

where $\$^{T}$ is the twist transpose, described as $\$^{T}=[P Q R L M N]$, where $[P Q R L M N]$ are the plücker coordinates and $G$ is the graminiam matrix given by

$$
G=\sum_{i=1}^{n} \$_{r, i} \cdot \$_{r, i}^{T} .
$$

The optimization problem constraint is the invariant norm which takes the frame-invariant screw portion magnitude [27]. It is defined as

$$
\begin{aligned}
\|\$\| & =\sqrt{\omega \cdot \omega} \\
& =\sqrt{\$^{T} D \$^{\prime}}
\end{aligned}
$$


where

$$
D=\left[\begin{array}{ll}
I_{(3 \times 3)} & 0_{(3 \times 3)} \\
0_{(3 \times 3)} & 0_{(3 \times 3)}
\end{array}\right] .
$$

For a pure translation case (i.e., $\omega=0$ ). the invariant norm is

$$
\|\$\|=\sqrt{v \cdot v}
$$

considering Equations (17) and (19). The optimization problem to measure closeness to direct singularity may be expressed as

$$
M(X)=\left\{\begin{array}{cc}
\min _{\$} & F(\$)=\$^{T} G \$ \\
h(\$)= & \$^{T} D \$-1=0 .
\end{array}\right.
$$

This constrained problem may be transformed into an unconstrained problem by means of a Lagrange function $(L)$, introducing one Lagrange multiplier for each constraint. Hence, the optimization problem may be expressed as

$$
\min _{\$, \lambda} L(\$, \lambda)
$$

where the Lagrange function $L$ is described as

$$
L=\$^{T} G \$+\lambda\left(\$^{T} D \$-1\right) .
$$

Differentiating the Lagrange function respect to $\lambda$

$$
\frac{\partial L(\$, \lambda)}{\partial \lambda}=\$^{T} D \$-1=0,
$$

and differentiating the Lagrange function with respect to $\$$ and using the fact that $D$ and $G$ are symmetrical yields

$$
\frac{\partial L(\$, \lambda)}{\partial \$}=(G-\lambda D) \$=0
$$

Note that $\partial L(\$, \lambda) / \partial \lambda$ is the optimization constraint exposed in Equation (22) while for $\partial L(\$, \lambda) / \partial \$$, the matrix expression in the parenthesis has to be singular for a non-trivial solution. In other words

$$
\operatorname{det}((G-\lambda D))=0
$$

The Equation (27) is the corresponding eigenvalue problem that may be rewritten as

$$
\operatorname{det}\left(\xi I-G^{-1} D\right)
$$

where $I_{(6 \times 6)}$ is a identity matrix and $\xi=1 / \lambda$ is the eigenvalue of $\left[G^{-1} D\right]$. Thus, the minimal function value is related to the minimal eigenvalue [27]. It may be proven rewriting Equation (25) as

$$
G \$=\lambda D \$
$$

Substituting Equation (29) into the objective function $(F)$ and using the constraint $h$

$$
F=\$^{T} G \$=\lambda \$^{T} D \$=\lambda
$$


Thus the minimization problem may be written as

$$
\min _{\$, \lambda} L(\$, \lambda)=\lambda_{\min } .
$$

\subsection{Geometrical Constraint}

The geometrical constraints occur due to collision between kinematic chains or due to violation of joint limits, which are specific for each joint according to its range of motion. These restriction types generally may be expressed explicitly.

\subsubsection{Prismatic Joint}

In this case the limitations are given by the joint sliding range (see Figure 3 ). The minimum length of $i$-th link is denoted by $\rho_{i m i n}$ and the maximum length by $\rho_{i m a x}$.

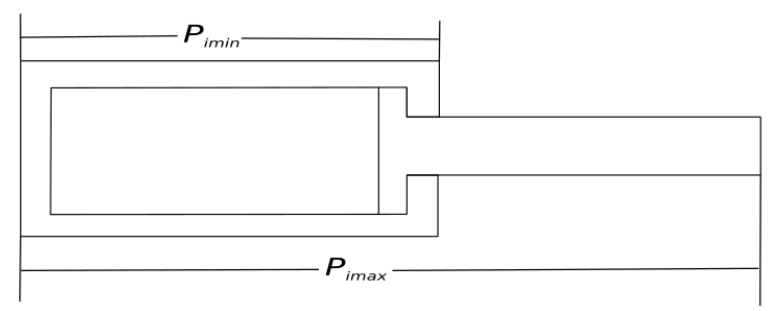

Figure 3. Prismatic Joint Mobility.

\subsubsection{Spherical Joint}

Spherical joint limitations may be represented through a cone which define the joint mobility or its range of motion (see Figure 4). In this case, the cone angle $\beta$ is the maximum misalignment angle of the joint (assumed to be less than $90^{\circ}$ ) and $\hat{\mathbf{j}}$ is the unit vector along the axis of symmetry [29].

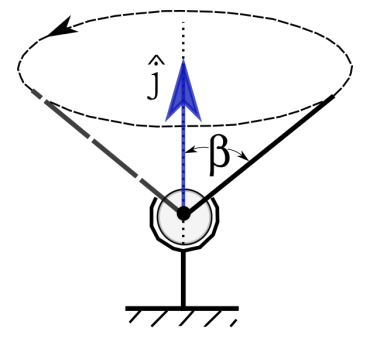

Figure 4. Spherical Joint Mobility.

\subsubsection{Kinematic Chain Collision}

The kinematic chain collision may be described as an intersection between two lines. It is important to notice that, the distance between these lines is given by their common normal line magnitude. Assume that the axes of two cylindrical segments (usual link geometry) $A_{j} B_{j}$ and $A_{j+1} B_{j+1}$ with radius $R_{j}$ and $R_{j+1}$ do not collide, if their common normal line norm follow this condition [30]:

$$
\operatorname{dist}\left(A_{j} B_{j}, A_{j+1} B_{j+1}\right) \geq R_{j}+R_{j+1}
$$

\section{Workspace Analysis}

In this section the 6 UPS orientation workspace is presented considering the kinematic and the geometric constraints mentioned above. In the proposed analysis the base radius $(r=1 \mathrm{~m})$, the platform radius $(R=0.5 \mathrm{~m})$ and a platform initial orientation $(\varphi=0, \vartheta=0, \psi=0)$ are regarded. 
The analysis is reduced to the achievable orientations in the plane $y x$ due to the complex geometry of the orientation workspace.

\subsection{Direct Singularity}

An algorithm in MATLAB is applied aiming at measuring the robot direct singularity closeness $\lambda$. So, the algorithm calculates the wrench value for each instantaneous PM position. Hesselbach et al. [31] determines that the direct singularity takes place when $\sqrt{\lambda}$ falls under 0.03 . Thus, the constraint equation for the direct kinematic singularity may be written as:

$$
\sqrt{\lambda_{\text {min }}}>0.03 \text {. }
$$

\subsection{Geometrical Constraints}

The mechanical constraints considered in this analysis are: (a) prismatic joint limit, (b) kinematic chain interference.

$$
\begin{gathered}
\rho_{\min } \leq l_{p i} \leq \rho_{\max }, \\
d_{i j} \geq D,
\end{gathered}
$$

where $l_{p i}$ is the motion range for the $i$ th prismatic joint shown in Figure 2. $\rho$ min $=0.7$ and $\rho$ max $=1.4229$ are the minimum and maximum lengths of actuated joints. $d_{i j}$ is the distance between the $i$-th link and the $j$-th link $(i \neq j) . D=0.03$ is the diameter of each link.

As the first study case, a fixed platform position $p=\left[\begin{array}{lll}0 & 0 & 0.5\end{array}\right] \mathrm{m}$ is considered in Figure 5 is shown the direct kinematic index value associated with each platform orientation $(0, \vartheta, \psi)$, where the infeasible regions are related to the blue regions while the remains regions are regarded feasibles, i.e., the index value is higher that 0.03 . Several existing feasible regions where the platform may move as shown in Figure 5, which means that travel between regions in the same plane is possible if an appropriate platform path or platform configuration is established. It is important to note that the term "platform configuration" is related to the different orientations that the platform achieves within the orientation workspace.

In addition to that direct singularity index, other indices that associate the geometrical constraints with the orientation workspace boundaries are established: prismatic joint $\left(\lambda_{p}\right)$, legs collision $\left(\lambda_{c}\right)$ and passive joints $\left(\lambda_{y}\right)$.

The prismatic joint index represents the higher proximity percentage from the sliding link effector positions to the physical joint limits. Where $100 \%$ means that the the joint reached its physical limit. This measure restricts the parallel robot orientation workspace approximately $70 \%$ in relation to the direct kinematic index, in the case of plane analysis to a central feasible region as shown in Figure 6. where the infeasible reigions are yellow, while the remains regions are regarded feasibles.

The leg collision index indicates how close the limbs are to impact with each other. This collision is considered when the index value is $100 \%$. For the planar study shown in Figure 7 the higher index is $50 \%$, it means that there are no limitations related to the collision index. In this case the collision index is limited by the prismatic joint indice and the direct kinematic index. Therefore the orientation workspace in the plane $\psi=0.0$ is initially restricted by these constraints. The collision analysis is always considered because the workspace could be limited in other planes or in the space, e.g., in the plane $\psi=0.3$ index value increase to $98 \%$ as shown Figure 8 .

The orientation workspace shown in Figure 9 exhibits different feasible regions (in green) in which the parallel robot operates without violating the kinematics and prismatics joint constraints, see Figure 10. To achieve major rotations in some specific directions, the platform may be within one of these green regions. 


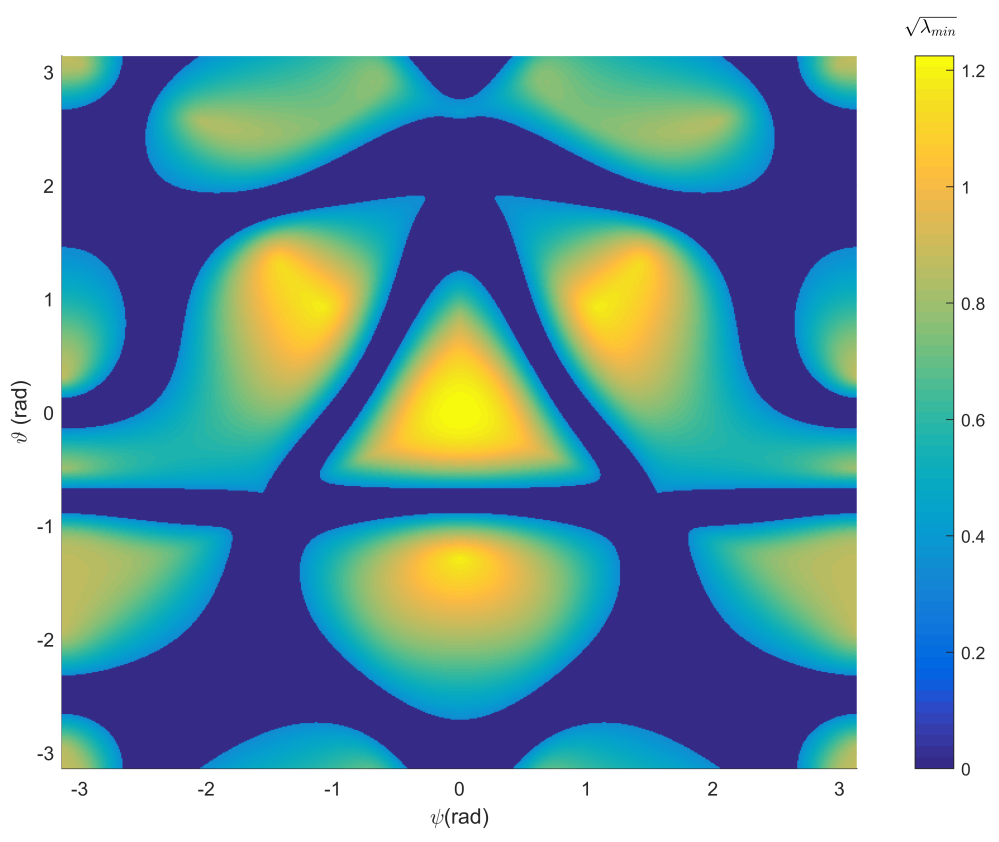

Figure 5. Orientation workspace associated with direct kinematic index $\sqrt{\lambda \min }$.

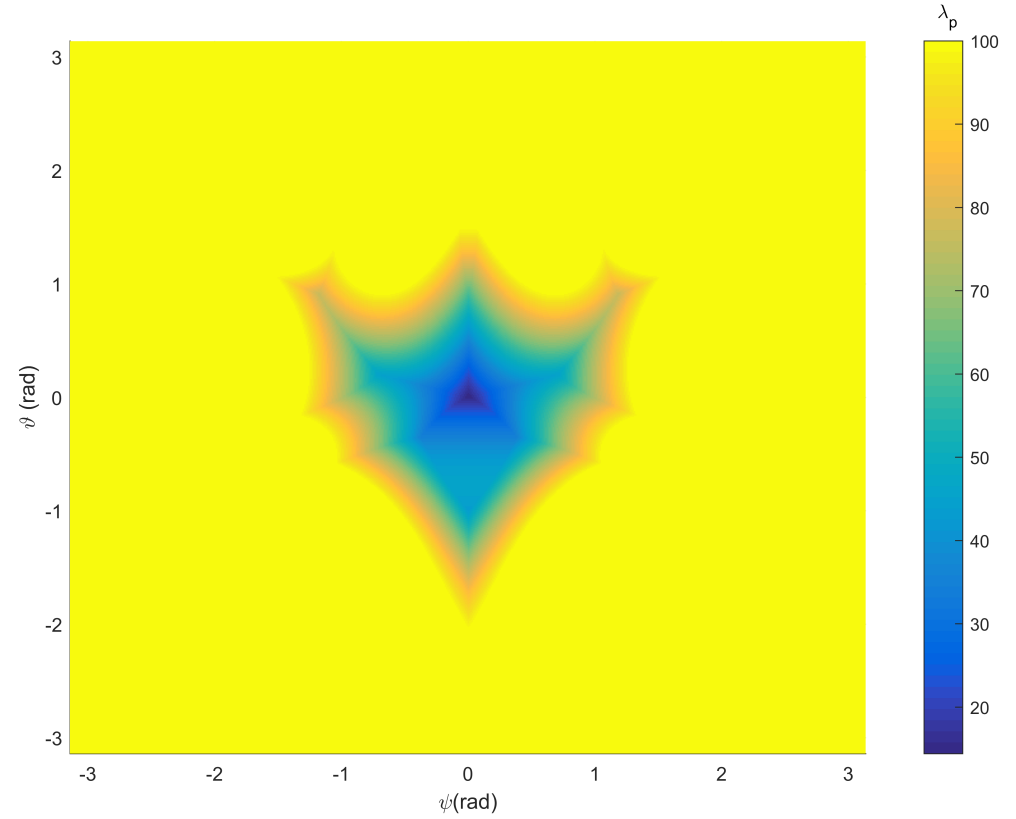

Figure 6. Orientation workspace associated with prismatic joint index. 


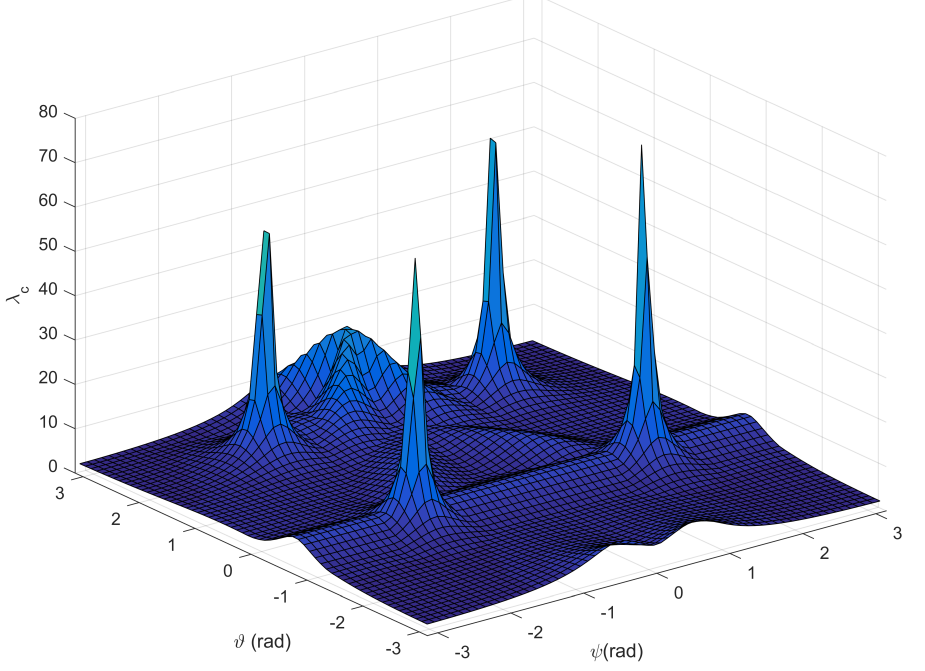

Figure 7. Orientation workspace associated with legs collision.

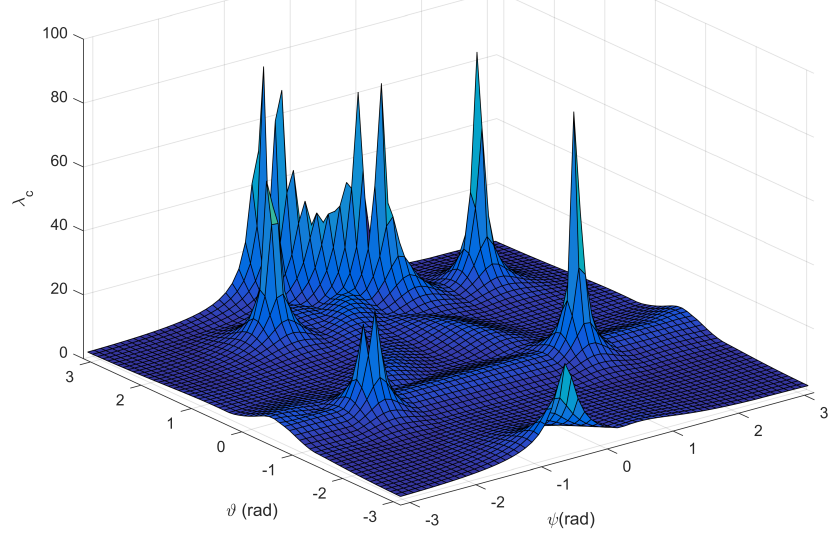

Figure 8. Orientation workspace associated with legs collision $\varphi=0.3$.

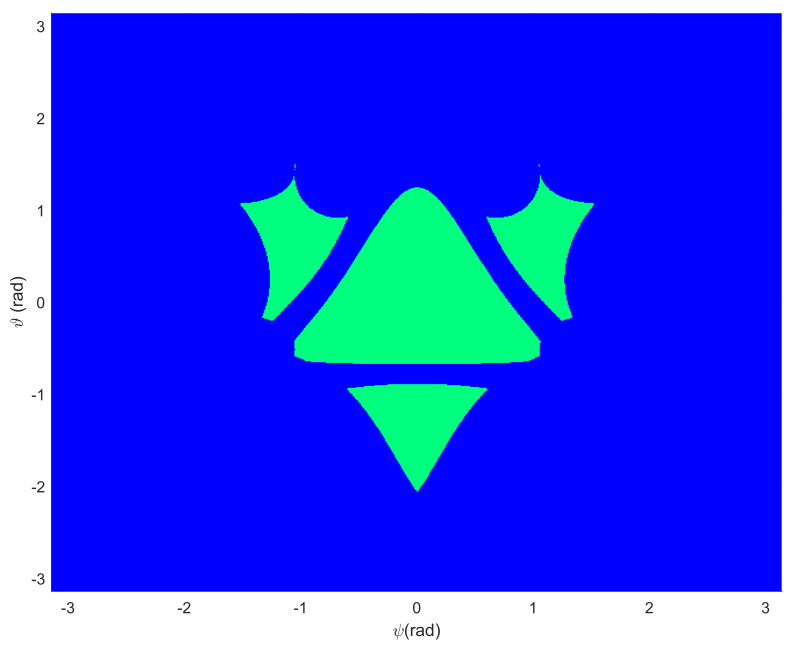

Figure 9. Orientation workspace without passive joint analysis. 


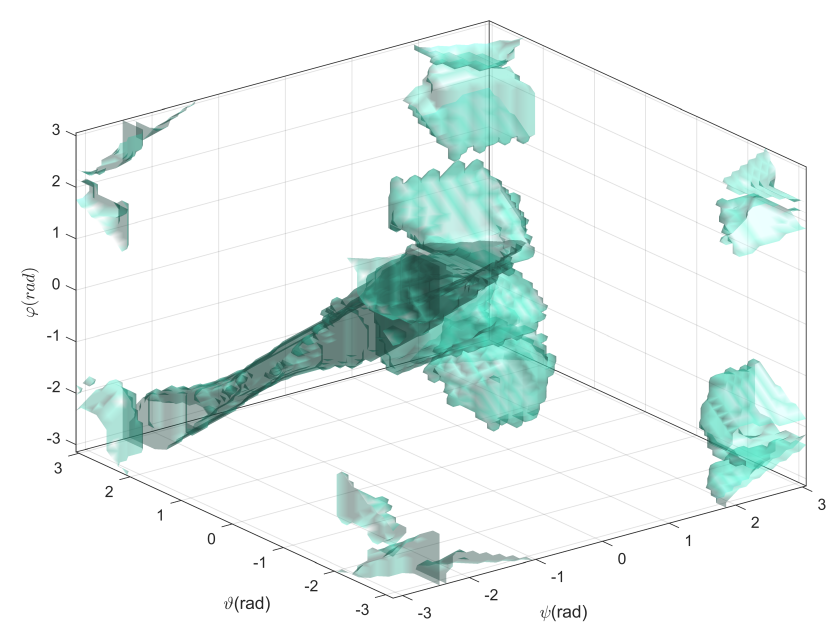

Figure 10. Three-dimensional orientation workspace without passive joint analysis.

The passive joint index is related to the non-actuated joint motion study, which presents physical restricted motion similar to the actuated joints (prismatics). In the S-G platform case, it refers to allowed movement in universal and spherical joints. The workspace related to passive joints is presented in Figure 11. Taking the passive joint analysis into account the orientation workspace is reduced to a unique feasible region (see Figure 12). In this case $\beta$ value is based on the Bonev researches [29].

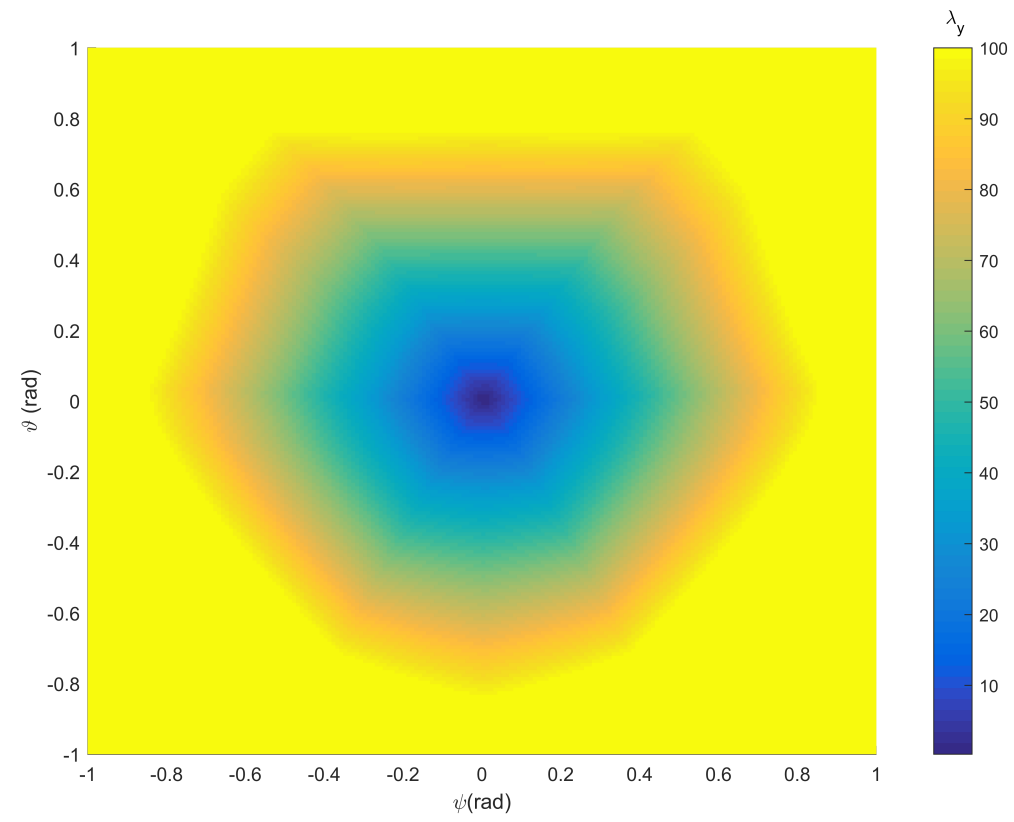

Figure 11. Orientation Workspace Associated to Passive Joint index with $\beta \leq 50^{\circ}$. 


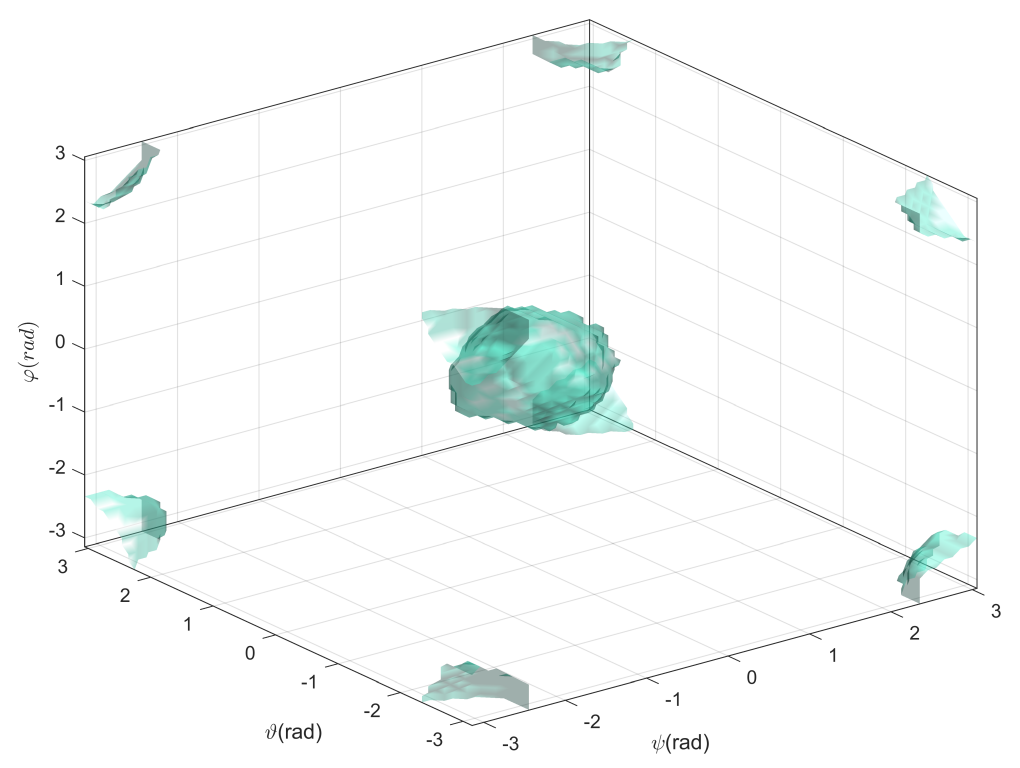

Figure 12. Three-Dimensional Orientation Workspace with passive Joints Analysis.

\section{Workspace Optimization}

In the present study, the workspace optimization aims at identifying the higher singularity free sphere also called the optimal sphere, which represents the reachable orientations by the platform in the three-dimensional space. However, due to the orientation workspace geometry, this task is complex. Thus, to find the optimal sphere, a genetic algorithm is implemented. Those algorithms are a robust type of evolutionary algorithms, which explore all the space avoiding fall in local minimums (see Figure 16). The optimization analysis takes into account the kinematic and geometric robot constraints.

\subsection{Genetic Algorithm (GA)}

Genetic algorithms are an increasingly popular method of optimization being applied to many fields, motivated by the "survival of the fittest" concept and the Darwin's theory of natural selection. Therefore, these algorithms use analogous processes as biological evolution to promote the best genes of a population. In the GA search process, only the function values are used to make progress toward a problem solution. The problem function differentiability is never required for the algorithm calculations. Therefore, it may be applied to all kinds of problems: discrete, continuous, and non-differentiable. For this reason the GAs are widely used in different practical engineering problems [32]. The algorithm starts by generating an initial population of random candidate solutions. Each individual in the population is then awarded a score based on its performance. For this purpose, the candidates are represented by binary strings and the GA population size is fixed [33]. The basic elements of natural genetics are reproduction, crossover and mutation, which are used in the genetic search procedure [12]. The optimization algorithm flowchart is shown Figure 13.

\subsubsection{Reproduction}

Reproduction operation is also called the selection operation because it selects good strings from the current population to form a mating pool. This operation is the first operation applied to the GAs population [12]. The reproduction operator is biased toward picking above-average strings of the current design set (population). Thus, multiple copies of better strings are inserted in the mating pool based on a probabilistic procedure. Usually, a string is selected from the mating pool with a probability proportional to its fitness, i.e., those with higher fitness should have a greater chance of selection [34]. 


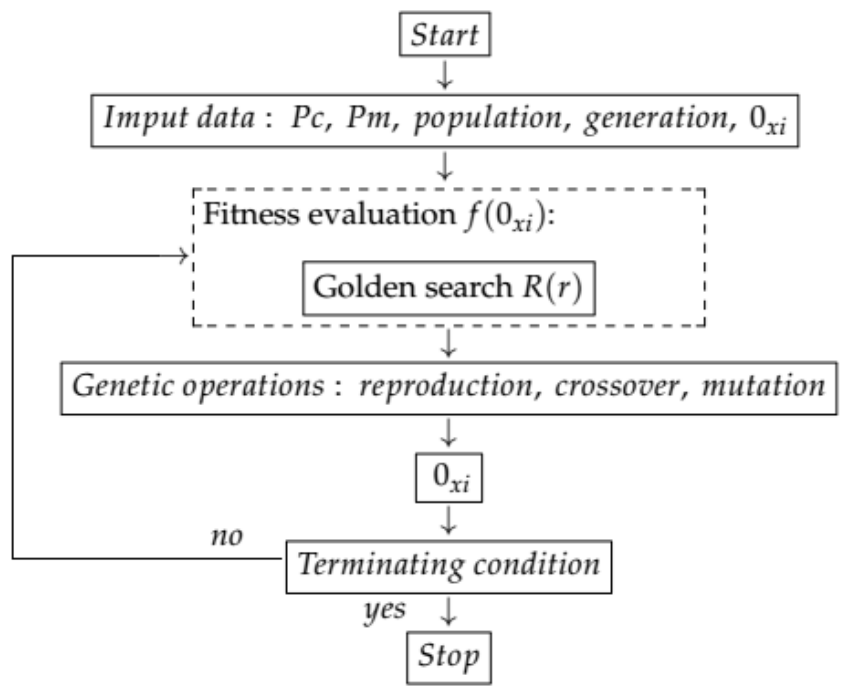

Figure 13. Genetic algorithm flowchart.

\subsubsection{Crossover}

Crossover is an operation that introduces variation into a population. It creates a new string by exchanging information among strings of the mating pool [12]. Thus, for the crossover operation two individual strings are selected, which are known as the parent string. These strings are picked at random from that mating pool generated by reproduction. Then, an information string portion is exchanged between the parents resulting in child strings [34]. To preserve some good strings for the next generation, a crossover probability, $p_{c}$, is used. Thus only $100 p_{c}$ of the strings in the mating pool will be used and $100\left(1-p_{c}\right)$ percent of the strings will be retained.

\subsubsection{Mutation}

Mutation introduces traits not in the original population, modifying a certain percentage of the bits in the list of chromosomes. So it keeps the GA from converging too fast before sampling the entire variable space. Therefore, the mutation operation allows the search outside the current region of variable space [35]. In practice, a mutation is applied to the new strings with a specific small mutation probability, pm. This operation modifies the binary digit 1 to 0 and vice versa.

\subsubsection{Objective Function}

The objective function, also called the fitness function, is related to the parameter to be maximized. In the present optimization analysis the parameter is the sphere radius, which is dependent on the sphere origin location. Therefore, to determine the optimal solution, the GA algorithm explore different origin positions into the orientation workspace i.e the origin sphere locations. However, for each origin position an optimal radius $(r)$ exists. In view of this, the optimization analysis is split in two parts. In the first part the maximal radius is determined by means of golden search procedure. While in the second part, the optimal sphere origin is identified by GAs. Thus, the fitness function is given by:

$$
f\left(o_{x 1}, o_{x 2}, o_{x 3}\right)=\operatorname{abs}\left(\min \left(R\left(o_{x 1}, o_{x 2}, o_{x 3}, r\right)\right)\right) .
$$

On the right side of Equation (36) the sphere radius $(r)$ is a variable and sphere origin coordinates $\left(o_{x 1}, o_{x 2}, o_{x 3}\right)$ are fixed values. Thus, the function $R(r)$ determines the feasible $(r)$ values associated with a specific sphere origin, whose value could range from 0 to $-r$ in the span $0 \leq r \leq \pi$, as shown in Figure 14. 


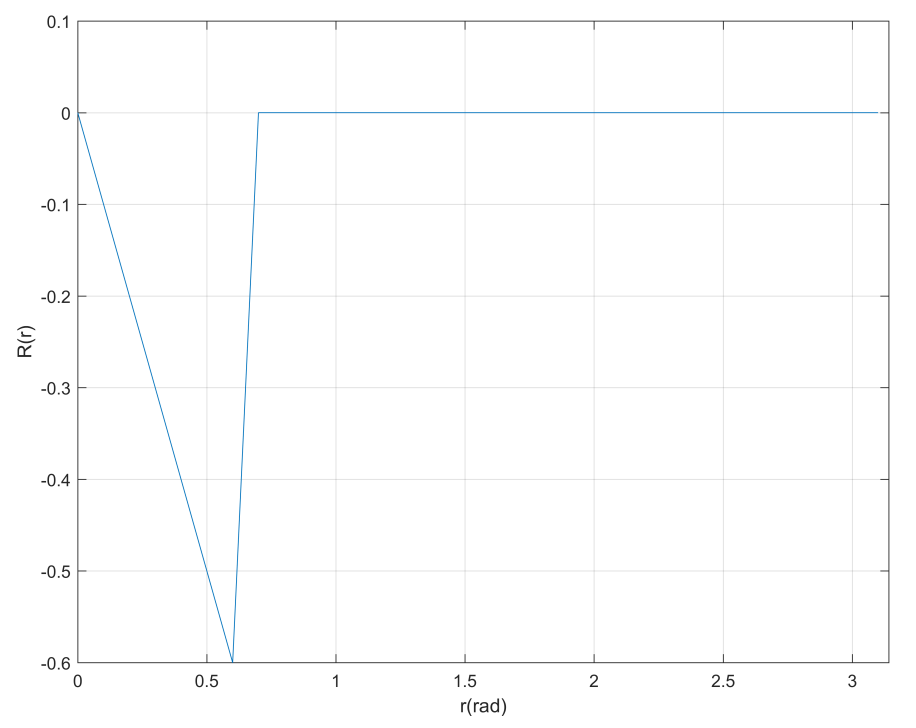

Figure 14. $R(x)$ Funcntion value with $o_{x 1}=0, o_{x 2}=0, o_{x 3}=0$ for a G-S platform.

\subsection{Optimization}

The optimization problem is described as:

$$
\begin{array}{cc}
\text { maximize } & f\left(0_{x i}\right) \\
\text { subject to }: & \beta \leq 50^{\circ} \\
& \sqrt{\lambda_{\min }}>0.03 . \\
\rho_{\min } \leq l_{p i} \leq \rho_{\max } \\
d_{i j} \geq D \\
-\pi \geq 0_{x i} \leq \pi
\end{array}
$$

where $0_{x i}=\left|0_{x 1}, 0_{x 2}, 0_{x 3}\right|$, the optimization contraints are associated with the kinematic and geometrical parallel robot charateristics mentioned below.

The objetive function convergence is shown in Figure 15. This function is associated with the best individual value of each generation. Due to the random GAs nature there is no specific rule to select the optimization algorithm operation parameters. Thus, they are selected based on previous attempts to find an optimal solution. The GA parameters are shown in Table 1.

Table 1. Genetic Algorithm Operation Parameters.

\begin{tabular}{cc}
\hline Population: & 80 \\
Generations: & 100 \\
Crossover $(P c):$ & 30 \\
Mutation $(P m):$ & 10 \\
\hline
\end{tabular}




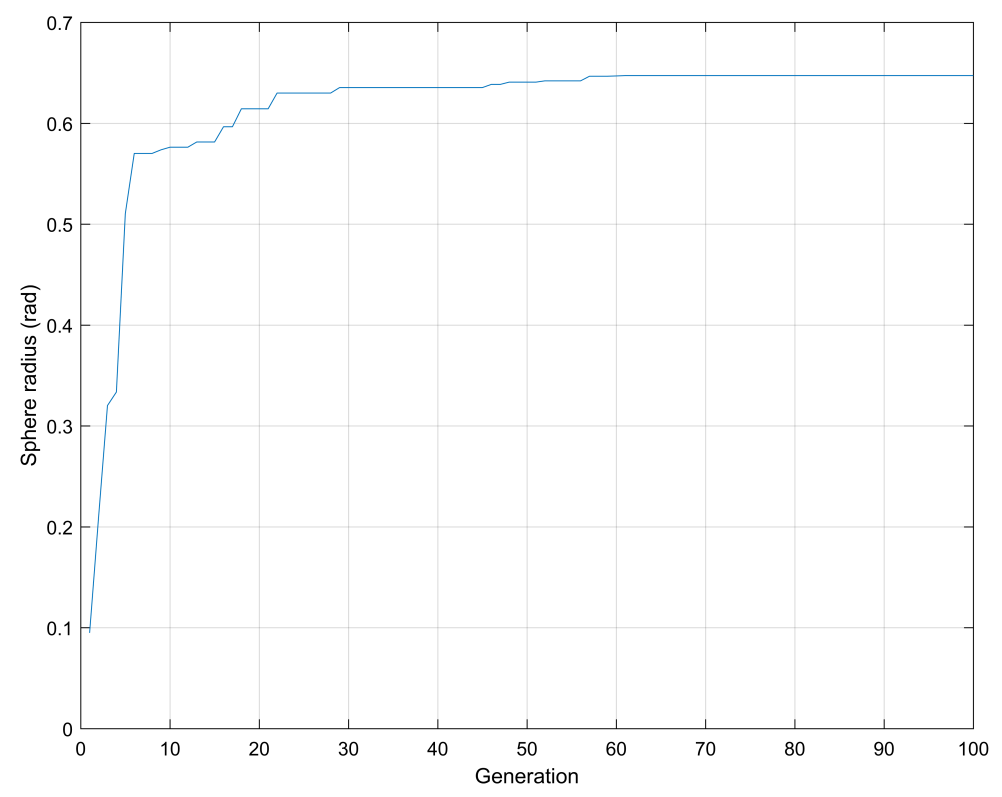

Figure 15. Objective function convergence.

The GA individuals explore all the space detecting feasible regions while the golden search modifies the sphere size. The individual convergences is shown in Figure 16. For this case the optimal solution is located at point $(-33,11,28) 10-4$. The maximal sphere within the orientation workspace is shown in Figure 17 and its radius is $R=0.646 \mathrm{rad}$. It means that the platform could reach these orientations $\theta_{x, y, z} \leq 0.646 \mathrm{rad}$ without falling into singularities.

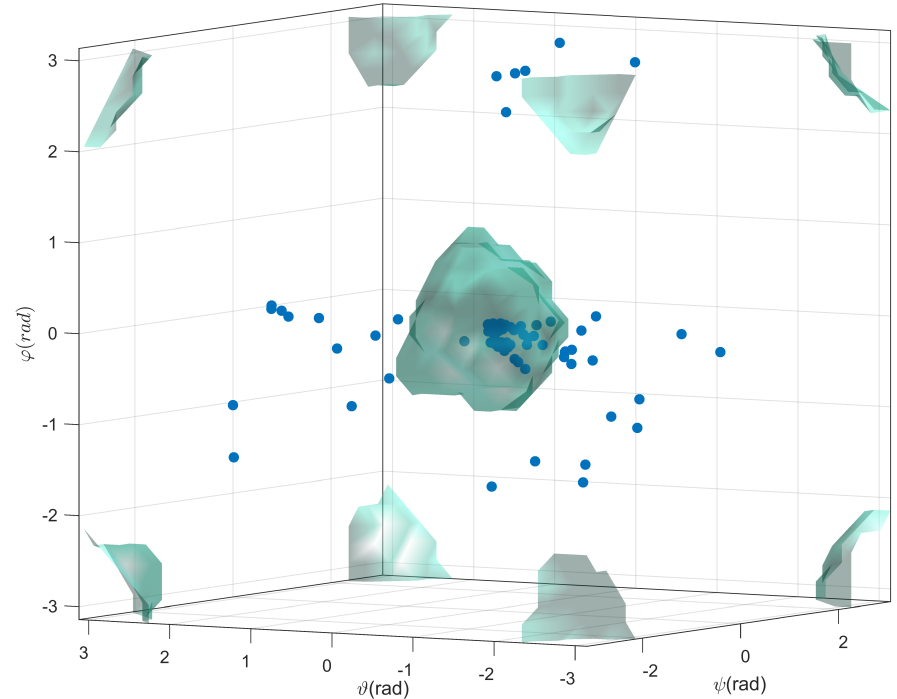

Figure 16. GAs individuals searching an optimal solution. 


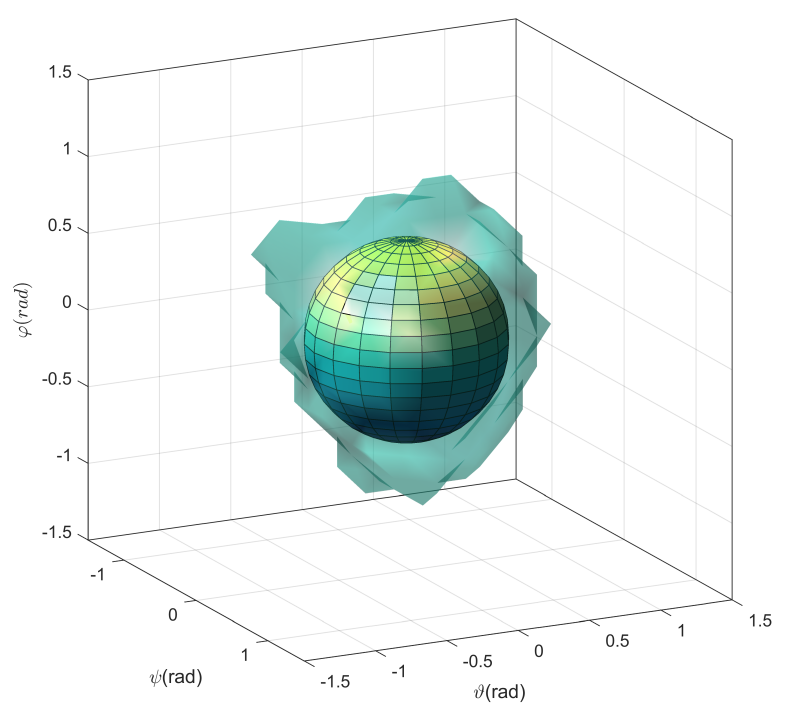

Figure 17. Maximal sphere within orientation workspace.

\section{Conclusions}

The main contribution of this work is to develop an optimization algorithm to establish the initial platform orientations, where the platform achieves a higher rotation in all directions. By taking into account the physical and kinematical constraints it is possible to know the workspace boundaries and its geometry. Due to the workspace complex geometry, the maximal platform orientations are approximated to a simplified geometry, i.e., sphere, where its origin is the initial platform orientation and the radius is the higher rotation magnitude that may be achieved in any direction. In the practice, this sphere may be named safety workspace.

In the workspace, different available regions may exist associated with the parallel robot kinematics and geometric characteristics where the platform may reach higher rotations as shown in Figures 9 and 10. These rotations are reduced due to passive joints mobility range. However, this disadvantage could be treated with a passive joint optimization, where the passive joint axis angle $\beta$ is the parameter to be optimized.

A method for solving the mobility problems for general parallel robot architecture based on screw theory analysis is provided. This method takes into account the Jacobian matrices and the actuator contributions on the platform motion. Furthermore, parallel robot physical constraints as geometrical limits are considered.

Author Contributions: L.G. develop an optimization algorithm to determine the maximal free-constraint sphere within the parallel robot workspace, which may be considered the safety region and A.C. supervised the investigation methodology.

Acknowledgments: The authors would like to express the following acknowledgments. To CAPES-BRAZIL, 272 TECNOLOGIA ASSISTIVA PGPTA 59/2014-3686/2014, for the financial support.

Conflicts of Interest: The authors declare no conflicts of interest.

\section{References}

1. Tsai, L.W. Robot Analysis: The Mechanics of Serial and Parallel Manipulators; John Wiley \& Sons: Hoboken, NJ, USA, 1999.

2. Gao, Z.; Zhang, D. Workspace representation and optimization of a novel parallel mechanism with three-degrees-of-freedom. Sustainability 2011, 3, 2217-2228. [CrossRef]

3. Pott, A. Efficient Computation of the Workspace Boundary, Its Properties and Derivatives for Cable-Driven Parallel Robots. In Computational Kinematics; Springer: Dordrecht, The Nertherlands, 2018; pp. 190-197. 
4. Bayani, H.; Masouleh, M.T.; Karimi, A.; Cardou, P.; Ebrahimi, M. On the determination of the maximal inscribed ellipsoid in the Wrench-Feasible Workspace of the cable-driven parallel robots. In Proceedings of the Second RSI/ISM International Conference on IEEE Robotics and Mechatronics (ICRoM), Tehran, Iran, 15-17 October 2014; pp. 422-427.

5. Monsarrat, B.; Gosselin, C.M. Workspace analysis and optimal design of a 3-leg 6-DOF parallel platform mechanism. IEEE Trans. Robot. Autom. 2003, 19, 954-966. [CrossRef]

6. Sun, T.; Song, Y.; Dong, G.; Lian, B.; Liu, J. Optimal design of a parallel mechanism with three rotational degrees of freedom. Robot. Comput. Integr. Manuf. 2012, 28, 500-508. [CrossRef]

7. St-Onge, B.M.; Gosselin, C.M. Singularity analysis and representation of the general Gough-Stewart platform. Int. J. Robot. Res. 2000, 19, 271-288. [CrossRef]

8. Kanaan, D.; Wenger, P.; Caro, S.; Chablat, D. Singularity analysis of lower mobility parallel manipulators using Grassmann-Cayley algebra. IEEE Trans. Robot. 2009, 25, 995-1004. [CrossRef]

9. Ben-Horin, P.; Shoham, M. Singularity condition of six-degree-of-freedom three-legged parallel robots based on grassmann-cayley algebra. IEEE Trans. Robot. 2006, 22, 577-590. [CrossRef]

10. Coste, M.; Moussa, S. On the rationality of the singularity locus of a Gough-Stewart platform-Biplanar case. Mech. Mach. Theory 2015, 87, 82-92. [CrossRef]

11. Karimi, A.; Masouleh, M.T.; Cardou, P. Singularity-free workspace analysis of general 6-UPS parallel mechanisms via convex optimization. Mech. Mach. Theory 2014, 80, 17-34. [CrossRef]

12. Rao, S.S.; Rao, S.S. Engineering Optimization: Theory and Practice; John Wiley \& Sons: Hoboken, NJ, USA, 2009.

13. Stan, S.D.; Manic, M.; Balan, R.; Maties, V. Genetic algorithms for workspace optimization of planar medical parallel robot. In Proceedings of the IEEE International Conference on Emerging Trends in Computing (ICETIC), Tamil Nadu, India, 8-10 January 2009; pp. 8-10.

14. Abbasnejad, G.; Daniali, H.M.; Kazemi, S. A new approach to determine the maximal singularity-free zone of 3-RPR planar parallel manipulator. Robotica 2012, 30, 1005-1012. [CrossRef]

15. Murray, R.M.; Li, Z.; Sastry, S.S.; Sastry, S.S. A Mathematical Introduction to Robotic Manipulation; CRC Press: Boca Raton, FL, USA, 1994.

16. Huang, Z.; Li, Q.; Ding, H. Theory of Parallel Mechanisms; Springer Science \& Business Media: Berlin, Germany, 2012; Volume 6.

17. Campos, A. Cinemática Diferencial De Manipuladores Empregando Cadeias Virtuais. Ph.D. Thesis, Universidade Federal de Santa Catarina, Florianópolis, Brazil, 2004.

18. Jayakrishna, S.R.; Babu, G.S. Dexterity Indices of 6-Ups Parallel Manipulator. Int. J. Mech. Eng. Technol. 2015, $6,1-8$.

19. Davidson, J.K.; Hunt, K.H. Robots and Screw Theory: Applications of Kinematics and Statics to Robotics; Oxford University Press on Demand: Oxford, UK, 2004.

20. Zhao, J.; Li, B.; Yang, X.; Yu, H. Geometrical method to determine the reciprocal screws and applications to parallel manipulators. Robotica 2009, 27, 929-940. [CrossRef]

21. Li, W.; Angeles, J. The design for isotropy of a class of six-dof parallel-kinematics machines. Mech. Mach. Theory 2018, 126, 34-48. [CrossRef]

22. Zlatanov, D.; Gosselin, C.M. Singularity analysis of 3-DOF planar parallel mechanisms via screw theory. ASME J. Mech. Des. 2003, 125, 573-581.

23. Li, H. The Analytical Singularity Locus Equation and the Determination of Singularity-Free Zones in the Workspace of the General Gough-Stewart Platform. Ph.D. Thesis, Université Laval, Quebec City, QC, Canada, 2005.

24. Li, W.; Angeles, J. Full-mobility 3-CCC parallel-kinematics machines: Forward kinematics, singularity, workspace and dexterity analyses. Mechan. Mach. Theory 2018, 126, 312-328. [CrossRef]

25. Merlet, J.P. Parallel Robots; Springer Science \& Business Media: Berlin, Germany, 2006; Volume 128.

26. Wang, X.; Zhang, D.; Zhao, C.; Zhang, H.; Yan, H. Singularity analysis and treatment for a 7R 6-DOF painting robot with non-spherical wrist. Mech. Mach. Theory 2018, 126, 92-107. [CrossRef]

27. Voglewede, P.A. Measuring Closeness to Singularities of Parallel Manipulators With Application to the Design of Redundant Actuation. Ph.D. Thesis, Georgia Institute of Technology, Atlanta, GA, USA, 2004.

28. Pottmann, H.; Peternell, M.; Ravani, B. Approximation in line space-Applications in robot kinematics and surface reconstruction. In Advances in Robot Kinematics: Analysis and Control; Springer: Berlin, Germany, 1998; pp. 403-412. 
29. Bonev, I.A.; Ryu, J. A new approach to orientation workspace analysis of 6-DOF parallel manipulators. Mech. Mach. Theory 2001, 36, 15-28. [CrossRef]

30. Kelaiaia, R.; Company, O.; Zaatri, A. Multiobjective optimization of a linear Delta parallel robot. Mech. Mach. Theory 2012, 50, 159-178. [CrossRef]

31. Hesselbach, J.; Bier, C.; Campos, A.; Lowe, H. Direct kinematic singularity detection of a hexa parallel robot. In Proceedings of the IEEE International Conference on IEEE Robotics and Automation (ICRA), Barcelona, Spain, 18-22 April 2005; pp. 3238-3243.

32. Arora, J. Introduction to Optimum Design; Elsevier: New York, NY, USA, 2004.

33. Weile, D.S.; Michielssen, E. Genetic algorithm optimization applied to electromagnetics: A review. IEEE Trans. Antennas Propag. 1997, 45, 343-353. [CrossRef]

34. McCall, J. Genetic algorithms for modelling and optimisation. J. Comput. Appl. Math. 2005, 184, $205-222$. [CrossRef]

35. Syahputra, R. Distribution Network Optimization Based on Genetic Algorithm. J. Electr. Technol. UMY 2017, 1, 1-9.

(C) 2018 by the authors. Licensee MDPI, Basel, Switzerland. This article is an open access article distributed under the terms and conditions of the Creative Commons Attribution (CC BY) license (http://creativecommons.org/licenses/by/4.0/). 